\title{
A rapid HPLC method for the determination of sulphonamides and trimethoprim in feed premixes
}

\author{
V. Goulas ${ }^{1}$, T. Anisimova Andreou², C. Angastinioti Moditi ${ }^{2}$ and O. Tzamaloukas ${ }^{1,3}$ \\ ${ }^{1}$ University of Technology, Department of Agricultural Sciences, Biotechnology and Food Science \\ Limassol, P.O Box 50329, Cyprus \\ 2 VitaTrace Nutrition Ltd, P.O Box 23886, Nicosia, Cyprus
}

KEY WORDS: additives, antibiotics, sulpha, medicated feed, feed premixes

Received: 12 July 2013

Revised: 26 February 2014

Accepted: 12 June 2014

${ }^{3}$ Corresponding author:

e-mail:ouranios.tzamaloukas@cut.ac.cy

\begin{abstract}
A simple HPLC method for the simultaneous determination of sulphadimidine, sulphadiazine, sulphamethoxazole and trimethoprim antibiotics in medicated feeds has been developed, validated, and applied to commercial feed premixes. Ultrasound-assisted extraction was used for the recovery of the studied antibiotics without the need for a further clean-up step. The sulphonamides and trimethoprim were separated on a Symmetry C18 (5 $\mu \mathrm{m}$, $4.6 \times 250 \mathrm{~mm}$ ) column using 10-min isocratic elution and UV detection at $254 \mathrm{~nm}$. The results showed that the method is robust with adequate recovery (minimum 95.19\%), satisfactory repeatability (1.39\%-2.69\%) and intermediate precision $(1.36 \%-4.16 \%)$, as shown by the validation assessment herein. The advantages of the proposed method are the speed, the ease of the clean-up protocol, and the low cost of the needed instrumentation, rendering it highly applicable to the feed industry.
\end{abstract}

\section{Introduction}

The use of medicated feeds (i.e. feedstuffs that include prescribed medicinal products) is the main administration method in intensive farming and the most effective way for a farmer to give medicine to his livestock (Turnidge, 2004). Mixes of sulphonamides (SAs) and trimethoprim (TMP) constitute the second most commonly used antimicrobials in authorized premixes in the European Union because of their broad spectrum antibacterial activity for the treatment of respiratory and/or gastrointestinal tract infections in sheep, cattle and pig production (EU Commission, 2010). Due to the awareness of the development and the spread of antibiotic resistance, however, alternatives to medicated feeds have been proposed (Link et al., 2005; Grashorn, 2010), while the legal framework of the EU relating to the use of antimicrobials in feeds is extremely rigorous (EU Commission, 2010). Therefore, the use of validated methods for the simultaneous determination of sulphonamides and trimethoprim in feed premixes is of high practical issue for different stakeholders in feed production, including the feed industry, inspection authorities, or even authorized farm mills.

Although several analytical methods are reported in the literature for determination of SAs, alone or in combination with TMP in different biological fluids (Teshima et al., 2004), foods (de Paula et al., 2008), or environmental samples (Cahill et al., 2004), only 
a limited number of analytical protocols for feeds has been published (Samanidou and Evaggelopoulou, 2008). The methods investigating feed premixes are mainly based on enzyme-linked immuneabsorbent assays, ELISAs (Jimenez et al., 2009), or high performance liquid chromatography methods, combined or not, with tandem mass spectrometry (Croubels et al., 2002; Boscher et al., 2010; Lopes et al., 2012). Nevertheless, the methods suggested to date for the simultaneous determination of SASand TMP require either a long and tedious analysis protocol (DeAngelis et al., 1990; Renew and Huang, 2004), or more explicit instrumentation, like an FTIR spectrometer (Silva et al., 2009), capillary electrophoresis system equipped with photodiode array detector (Farooq et al., 2009), or LC-MS/MS set-up (Boscher et al., 2010; Lopes et al., 2012).

The scope of the present study was to develop and validate a straightforward, low-cost and simple HPLC method for the simultaneous quantification of sulphadimidine (SDM), sulphadiazine (SDZ), sulphamethoxazole (SMZ) and TMP for routine analysis of feed premixes. Furthermore, time-consuming and laborious pre-treatment procedures were to be avoided in order to propose a widely applicable routine analysis method.

\section{Material and methods}

Chemicals. Sulphadiazine (4-amino-N-pyrimidin-2-N-2-ylbenzesulphonamide), sulphamethoxazole (4-amino-N-(5-methyl-3-iso-xazolyl) benzenesulphonamide), sulphadimidine (4-amino-N-(4,6dimethylpyrimidin-2-yl) benzenesulphonamide), and trimethoprim (5-(3,4,5-trimethoxybenzyl) (pyrimidine-2,4-diamine) were obtained from Sigma Aldrich (Figure 1). Triethylamine and glacial acetic were obtained from Sigma-Aldrich (St. Louis, MO). Acetonitrile and water were HPLC grade and provided by Scharlau (Barcelona, Spain). Different commercial feed premixes were kindly offered by Vita Trace Nutrition Ltd.

Sample extraction. The extraction of the premixes was done in triplicate for each sample as follows: $200 \mathrm{mg}$ of premix sample was mixed with $50 \mathrm{ml}$ methanol in an ultrasonic bath for $20 \mathrm{~min}$ at room temperature. Then, the extracts were filtered through a $0.20 \mu \mathrm{m}$ PTFE filter (Waters, Milford, MA) before injection into the HPLC.

Instrumentation. The HPLC system consisted of a binary DIONEX P680 pump equipped with a Dionex DG 1310 Degasser. The samples were injected using a Dionex ASI-100 autosampler.

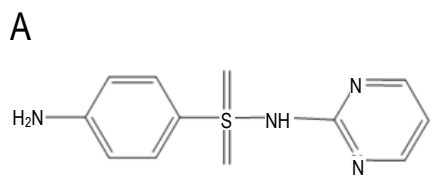

B

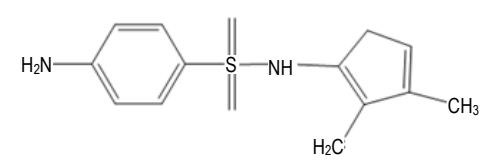

C
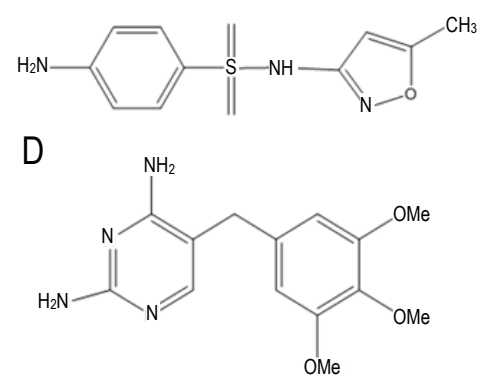

Figure 1. Chemical structures of sulphadimidine $(A)$, sulphadiazine (B), sulphamethoxazole (C) and trimethoprim (D)

Antibiotic monitoring was accomplished at $254 \mathrm{~nm}$ using a diode array (DAD) system Dionex UVD $340 \mathrm{U}$.

Chromatographic conditions. The chromatographic separation was carried out on a Symmetry C18 $(5 \mu \mathrm{m}, 4.6 \times 250 \mathrm{~mm})$ column. Two litres of the mobile phase were prepared by mixing water:acetonitrile:triethylamine $(1400: 400: 2, \mathrm{v} / \mathrm{v} / \mathrm{v})$. The $\mathrm{pH}$ of the elution solvent was adjusted to 5.9 using glacial acetic acid and the solution was diluted to 21 with HPLC-grade water as described for the determination of SMZ and TMP in a previous study for pharmaceutical tablets (Silva et al., 2009). A flow rate of $1.7 \mathrm{ml} \cdot \mathrm{min}^{-1}$ was used for the chromatographic separation.

The accuracy of the method is presented in terms of trueness and recovery. The trueness was determined by preparing and measuring six different samples from each of the raw material samples (SDM, SDZ, SMZ and TMP). The results were used to calculate the $\mathrm{SD}$, relative standard deviation (RSD) and trueness. Recovery was determined by using real samples containing known amounts of the analytes. Five different samples were prepared containing $80 \%, 90 \%, 100 \%, 110 \%$ and $120 \%$ of each analyte, respectively. Three injections were made for each different concentration on the same day. Recovery was calculated from these results.

The precision of the method was determined in terms of repeatability and intermediate precision. A sample containing $100 \%$ of each analyte was used 
for the determination of these terms. Repeatability was determined by preparing and measuring six different samples from the real sample within short time intervals. Two injections were made for each of six samples. From results the SD, RSD and the repeatability limit were calculated. The intermediate precision was determined by preparing and measuring a total of twelve different samples from the real samples by two different analysts (6 samples each) on different days. Two injections were made for each of the 12 samples. From these results, SD, RSD and intermediate precision were calculated.

Linearity was determined by measuring five different samples containing $80 \%, 90 \%, 100 \%, 110 \%$ and $120 \%$ of each analyte. From the results, correlation coefficient and R-square values were calculated.

\section{Results and discussion}

The extraction of SAs and TMP from feed premixes or other animal feedstuffs is a crucial step of the overall analytical process that leads to the quantification of targeted antibiotics. Recently, Sun et al. (2007) suggested that polar solvents are more suitable for the extraction of sulphonamides from natural animal casings than organic solvents such as dichloromethane, acetone, acetonitrile and ethyl acetate. Methanol has also been used for determination of
TMP in wastewaters (McClure and Wong, 2007). In the present study, the ability of various polar solvents such as water, methanol, ethanol and hydroalcoholic mixtures such as methanol:water (50:50) and ethanol:water (50:50) to extract the studied antibiotics was tested. Methanol had the highest recovery of antibiotics compared with the other studied solvents. The solubility of SDZ is low in ethanol, while the mixture of ethanol:water (50:50) gives high extractability of antibiotics without adequate selectivity.

The extraction time was also optimized during validation of the proposed method. It was observed that the recovery of the antibiotics was increased by extending the extraction time to $20 \mathrm{~min}$. Additional extraction time, e.g., up to 30 or $60 \mathrm{~min}$, did not further improve the recovery rate of the substances. The extraction of SAS by mixing feed premix sample with methanol using an ultrasonic bath for $10 \mathrm{~min}$ was around $90 \%$ of the spiking substance, but the repeatability of the extraction was unacceptable. Therefore, ultrasoundassisted extraction with methanol for $20 \mathrm{~min}$ was proposed since optimum recovery and satisfactory repeatability had been obtained. Furthermore, the proposed extraction protocol was a rapid, simple, and low-cost method without necessitating a solid phase extraction step as the previously reported methods (Boscher et al., 2010; Kumar and Companyo, 2011; Liu et al., 2011).

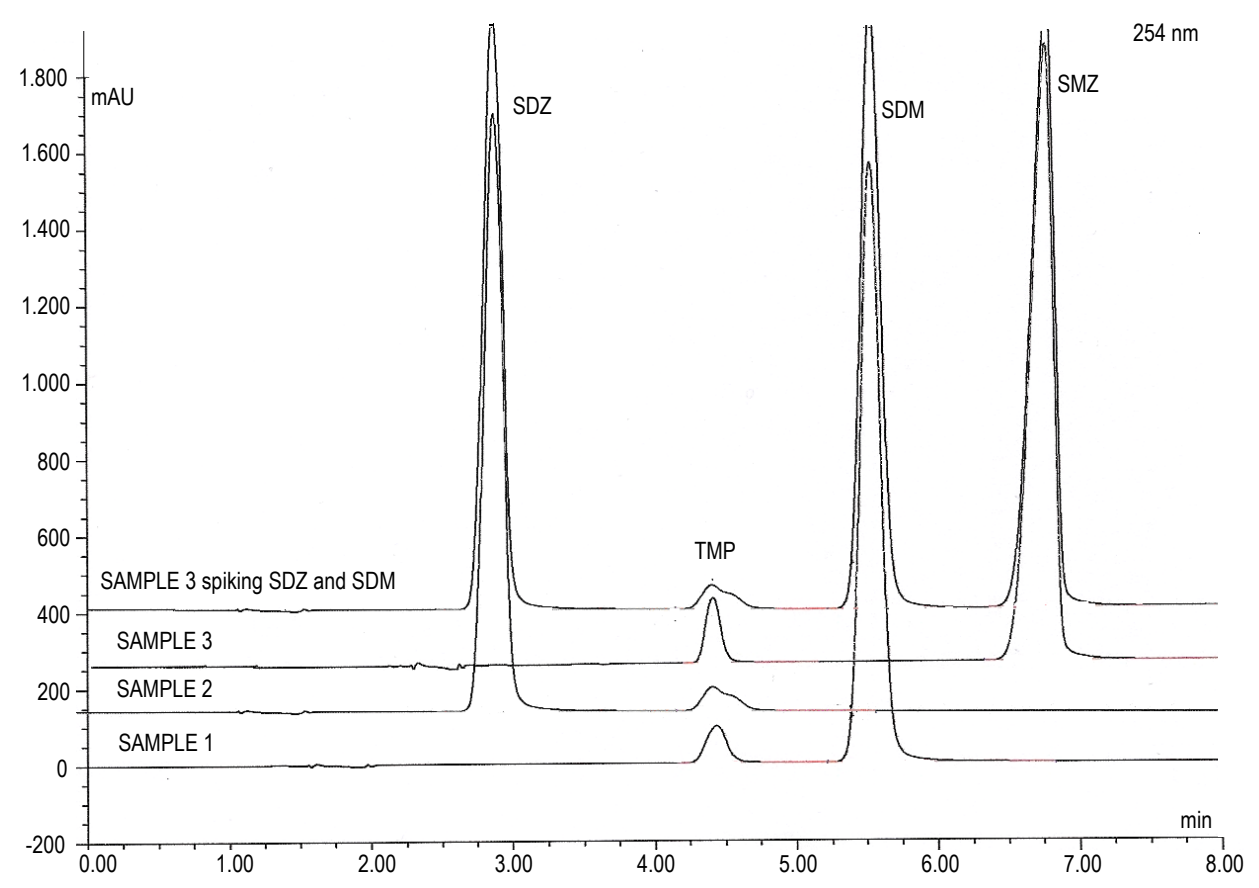

Figure 2. Chromatographic separation of commercial premixes. The chromatogram is recorded at $254 \mathrm{~nm}$ and the peaks under study are denoted 
The next step was to establish the chromatographic separation of studied antibiotics. Figure 2 shows that the proposed method allows successful separation of different antibiotics in a single run. The duration of chromatographic separation was less than eight min. The limits of detection and quantification were $3 \mu \mathrm{g} \cdot \mathrm{ml}^{-1}$ and $10 \mu \mathrm{g} \cdot \mathrm{ml}^{-1}$ for each antibiotic, respectively. The linear correlation between peak area and concentration of four compounds was determined. Data for five solutions of different concentrations in the range between 0.72 to $1.20 \mathrm{mg} \cdot \mathrm{ml}^{-1}$ for SDM, 0.80 to $1.19 \mathrm{mg} \cdot \mathrm{ml}^{-1}$ for $\mathrm{SDZ}, 0.59$ to $0.84 \mathrm{mg} \cdot \mathrm{ml}^{-1}$ for $\mathrm{SMZ}$, and 0.16 to $0.24 \mathrm{mg} \cdot \mathrm{ml}^{-1}$ for TMP were collected. The slope, intercept, and correlation coefficient were calculated and the results are listed in Table 1.

Table 1. Linearity of method for the determination of sulphonamides and trimethopsinin premixes

\begin{tabular}{|c|c|c|c|c|}
\hline Characteristics & $\begin{array}{l}\text { Sulpha- } \\
\text { diazine }\end{array}$ & $\begin{array}{l}\text { Sulpha- } \\
\text { methoxazole }\end{array}$ & $\begin{array}{l}\text { Sulpha- } \\
\text { dimidine }\end{array}$ & $\begin{array}{l}\text { Trime- } \\
\text { thoprim }\end{array}$ \\
\hline Slope & 5.78 & 5.19 & 5.72 & 1.84 \\
\hline Intercept & 0.0256 & 0.0014 & 1.09 & 0.0006 \\
\hline SD of intercept & 0.0233 & 0.0012 & 1.17 & 0.0007 \\
\hline $\begin{array}{l}\text { Correlation } \\
\text { coefficient }\end{array}$ & 0.9999 & 0.9999 & 0.9998 & 0.9999 \\
\hline
\end{tabular}

Table 2. Accuracy and precision of method for the determination of sulphonamides and trimethopsinin premixes

\begin{tabular}{|c|c|c|c|c|}
\hline \multirow[b]{2}{*}{ Antibiotic } & \multicolumn{2}{|l|}{ Accuracy } & \multicolumn{2}{|l|}{ Precision } \\
\hline & trueness & recovery & repeatability & $\begin{array}{l}\text { intermediate } \\
\text { precision }\end{array}$ \\
\hline Sulphadiazine & $99.77 \pm 0.89$ & $95.19-100.59$ & $2.59 \%$ & $4.16 \%$ \\
\hline $\begin{array}{l}\text { Sulpha } \\
\text { methoxazole }\end{array}$ & $99.70 \pm 1 ., 22$ & $95.74-99.26$ & $2.69 \%$ & $2.31 \%$ \\
\hline $\begin{array}{l}\text { Sulphadimi } \\
\text { dine }\end{array}$ & $99.08 \pm 2.63$ & $98.28-99.92$ & $1.35 \%$ & $1.36 \%$ \\
\hline Trimethoprim & $99.72 \pm 1.22$ & $95.44-98.68$ & $1.39 \%$ & $1.60 \%$ \\
\hline
\end{tabular}

The accuracy of the present method was tested by determining the recovery of the antibiotics under study. Five different premixes were prepared containing $80 \%, 90 \%, 100 \%, 110 \%$ and $120 \%$ of each analyte and their recovery was calculated (Table 2). The recovery ranged from $95.19 \%$ to $100.59 \%$ for sulphonamides and from $95.44 \%$ to $98.68 \%$ for trimethoprim. This recovery was comparable with those in previous studies in which an additional solid phase extraction step was used (Boscher et al., 2010; Liu et al., 2011). These findings confirmed that the proposed method is accurate with satisfactory repeatability (relative standard deviation of six replicates). The RSD of sulphadimidine and TMP was $1.35 \%$ and the corresponding value for sulphadiazine and sulphamethoxazole was 2-fold lower
(2.59\%-2.69\%). Boscher et al. (2010) achieved repeatability that ranged between $86.0 \%$ and $91.0 \%$ for trimethoprim and $86.9 \%$ to $91.2 \%$ for sulphadiazine, respectively.

\section{Conclusions}

In summary, the developed RP-HPLC method was found to be suitable for the separation and quantitative determination of sulphonamides and trimethoprim in medicated premixes or animal feeds. The use of ultrasound-assisted extraction in combination with methanol gave efficient recovery of antibiotics. The proposed assay is straightforward, simple, and applicable to routine analysis, while its accuracy and precision are particularly satisfactory and comparable with more complicated protocols.

\section{References}

Boscher A., Guignard C., Pellet T., Hoffmann L., Bohn T., 2010. Development of a multi-class method for the quantification of veterinary drug residues in feedingstuffs by liquid chromatography-tandem mass spectrometry. J. Chromatogr. A 1217 , 6394-6404

Cahill J.D., Furlong E.T., Burkhardt M.R., Kolpin D., Anderson L.G., 2004. Determination of pharmaceutical compounds in surfaceand ground-water samples by solid-phase extraction and highperformance liquid chromatography-electrospray ionization mass spectrometry. J. Chromatogr. A 1041, 171-180

Croubels S., Wassink P., De Backer P., 2002. Simultaneous determination of sulfadiazine and trimethoprim in animal feed by liquid chromatography with UV and tandem mass spectrometric detection. Anal. Chim. Acta 473, 183-194

de Paula F.C.C.R., de Pietro A.C., Cass Q.B., 2008. Simultaneous quantification of sulfamethoxazole and trimethoprim in whole egg samples by column-switching high-performance liquid chromatography using restricted access media column for on-line sample clean-up. J. Chromatogr. A 1189, 221-226

DeAngelis D.V., Wooley J.L., Sigel C.W., 1990. High-performance liquid chromatographic assay for the simultaneous measurement of trimethoprim and sulfamethoxazole in plasma or urine. Ther. Drug Monit. 12, 382-392

European Commission, 2010. Evaluation of the EU Legislative Framework in the Field of Medicated Feed. Food Chain Evaluation Consortium (Editors). Final Report, Brussels, pp. 188

Farooq M.U., Su P., Yang Y., 2009. Applications of a novel sample preparation method for the determination of sulfonamides in edible meat by CZE. Chromatographia 69, 1107-1111

Grashorn M.A., 2010. Use of phytobiotics in broiler nutrition - An alternative to infeed antibiotics? J. Anim. Feed Sci. 19, 338-347

Jiménez V., Companyó R., Guiteras J., 2009. Preparation of quality control materials for the determination of sulfonamides in animal feed. Food Addit. Contam. A 26, 969-977

Kumar P., Companyo R., 2011. Development and validation of an LCUV method for the determination of sulfonamides in animal feeds. Drug Test. Anal. 4, 368-375

Link R., Kovác G., Pistl J., 2005. A note on probiotics as an alternative for antibiotics in pigs. J. Anim. Feed Sci. 14, 513-519 
Liu R., He P., Li Z., Li R., 2011. Simultaneous determination of 16 sulfonamides in animal feeds by UHPLC-MS-MS. J. Chromatogr. Sci. 49, 640-646

Lopes R.P., De Freitas Passos T.E., De Alkimim Filho J.F., Vargas E.A., Augusti D.V., Augusti R., 2012. Development and validation of a method for the determination of sulfonamides in animal feed by modified QuEChERS and LC-MS/MS analysis. Food Control 28, 192-198

McClure E.L., Wong C.S., 2007. Solid phase microextraction of macrolide, trimethoprim, and sulfonamide antibiotics in wastewaters. J. Chromatogr. A 1169, 53-62

Renew J.E., Huang C.-H., 2004. Simultaneous determination of fluoroquinolone, sulfonamide, and trimethoprim antibiotics in wastewater using tandem solid phase extraction and liquid chromatography-electrospray mass spectrometry. J. Chromatogr. A 1042, 113-121
Samanidou V.F., Evaggelopoulou E.N., 2008. Chromatographic analysis of banned antibacterial growth promoters in animal feed. J. Sep. Sci. 31, 2091-2112

Silva F.E.B., Ferrão M.F., Parisotto G., Müller E.I., Flores E.M.M., 2009. Simultaneous determination of sulphamethoxazole and trimethoprim in powder mixtures by attenuated total reflection-Fourier transform infrared and multivariate calibration. J. Pharmaceut. Biomed. Anal. 49, 800-805

Sun H., Ai L., Wang F., 2007. Quantitative analysis of sulfonamide residues in natural animal casings by HPLC. Chromatographia $66,333-337$

Teshima D., Otsubo K., Makino K., Itoh Y., Oishi, R., 2004. Simultaneous determination of sulfamethoxazole and trimethoprim in human plasma by capillary zone electrophoresis. Biomed. Chromatogr. 18, 51-54

Turnidge J., 2004. Antibiotic use in animals - Prejudices, perceptions and realities. J. Antimicrob. Chemother. 53, 26-27 